\title{
PREDICTION OF PM2.5 CONCENTRATIONS USING TEMPERATURE INVERSION EFFECTS BASED ON AN ARTIFICIAL NEURAL NETWORK
}

\author{
R.A. Bahari, R. Ali Abbaspour, P. Pahlavani \\ Department of Surveying and Geomatics Eng., Engineering College, University of Tehran, Tehran, Iran \\ \{bahariamin, abaspour, pahlavani\}@ut.ac.ir,
}

KEY WORDS: PM2.5, Air pollution, Artificial Neural Network, Temperature inversion

\begin{abstract}
:
Today, air pollutant is a big challenge for busy and big cities due to its direct effect on both human health and the environment. Tehran, as the capital city of Iran, concludes 12 million people and is one of the most polluted cities in Iran. According to the reports, the main cause of Tehran's pollution is particle matters. The main factors affecting the density and distribution of pollution in Tehran are topography, traffic, and meteorological parameters including wind speed and direction, environment temperature, cloud cover, relative humidity, the sunshine overs a day, the rainfall, pressure, and temperature inversion. To help the urban management of Tehran, in this paper, a novel method is proposed to predicted PM2.5 concentration for upcoming 72 hours. The results show that the proposed model has high capability in predicting PM2.5 concentration and the achieved statistic coefficient of determination $\left(\mathrm{R}^{2}\right)$ was equal to 0.61 0.79 , which indicates the goodness of fit of our proposed model supports the prediction of PM2.5 concentration.
\end{abstract}

\section{INTRODUCTION}

There are many air pollutants such as carbon monoxide (CO), PM2.5, $\mathrm{PM} 10, \mathrm{SO}_{2}, \mathrm{NO}_{2}$, and ozone $\left(\mathrm{O}_{3}\right)$, which are adversely affecting human health in the polluted air. The high concentration of these pollutants is life-threatening and causes difficulties in breathing, headache, and dizziness in short time, as well as the different cancers and heart attacks in long time (Anikender Kumar, 2011).

Tehran as one of the most populated cities in the world is affected with the generally high rates of air pollutants. It seems that prediction models for air pollutants are appropriate tools to alarm the citizens. Moreover, it would be possible to plan suitable and low cost strategies for managing the pollution of Tehran using these models. Technically, there are different approaches for prediction. In this regard, artificial neural networks, which enable mapping the complex nonlinear models seem to be a suitable method to predict the quality parameters of air pollution in urban environments. The purpose of this study is preparation of an environmental warning system in the critical situations of air pollutions.

By using of capabilities of the neural network and the use of affective parameters such as temperature, wind speed, wind direction, relative humidity, and cloud cover, this paper has predicted the PM2.5 concentration for 72 hours later. In Tehran, one of the major causes of pollution affecting density is temperature inversion, which occurs in about $70 \%$ during the year. In former paper, this issue has received less attention, although, has a great influence on the density of atmospheric concentrations at low levels. The inversion strength is a suitable parameter for measuring the effect of the inversion on the pollution concentration that was imported into the proposed neural network in this paper.

\section{RELATED WORKS}

Many researches concerning prediction of air pollutions inside and outside the country have been done so far.

Ibarra Brastegi et al (2008), predicted the air pollution for a short period in Spain, using the data of air pollution, traffic, and meteorological as inputs of artificial neural network. Comparison the values predicted by the model with the actual ones clear that the artificial neural network is a powerful method for modelling the air pollution parameters and values simulated by the model have a great adaptation by the observed values. In order to predict PM10 concentration, Surendra Roy (2012) used a multi-layer perceptron neural network with three hidden layer and meteorological data. Result indicates that the artificial neural network has a great capability in predicting air pollution and the values resulted from the model have a great correlation to the values observed in air quality station. Boudaghpour (2011) used air pollution data from Bazar station between 2001 and 2006 to predict of NOx concentration in Tehran city. He used regression and time series to indicate the inputs of neural network. According to this model, the NOx presentation concentration has a correlation to 7 days ago gas concentration. The result showed that the model presented has a great capability to predict the air pollution of Tehran city. Khazaee et al (2011) modelled and predicted the concentration using a fuzzy model and used air quality station data of Tehran city by attention to RMSE values. Hatefi (2008) predicted the CO concentration for 4 days later using the meteorological and $\mathrm{CO}$ via the data mining and multilayer perceptron neural network. Finally, the results showed that GIS and data mining can be utilized as a dependable system for environmental warning.

\section{STUDY AREA AND DATA}

\subsection{STUDY AREA}

Tehran (Figure 1) by the area more than 70,000 hectares is located at southern Alborz sub-mountain. The average elevation of Tehran is about $1190 \mathrm{~m}$ above the sea level. Tehran from the north and east is ended to the elevations and just to the south is ended to the open plains. According to statistics, in 2011, this city had a population around 12 million. The city of meteorology climate is dry and arid. The Tehran average annual temperature is $17{ }^{\circ} \mathrm{C}$. The wind direction in Tehran in the cold season is from west to the east and in the warm season is from the south to the north and from the south west to the north east 


\subsection{DATA}

In this study, we used PM2.5 concentrations during the years 2012 to 2013 of Razi air pollution station (Figure 1). For the meteorological data, we used the data such as temperature, wind speed, wind direction, cloud cover, relative humidity acquired by Mehrabad synoptic station for the years mentioned above. The upper atmosphere information of Mehrabad station with the same chart and Skew-t graph for two hours (0\&12 UTC) was achieved by the University of Wyoming website. This is because the upper atmospheric data was available in these two hours (0\&12 UTC). Hence, we should prepare the inversion information in these special hours in addition to the other information such as PM2.5 concentration and meteorological data, including temperature, wind speed and direction, relative humidity, and cloud cover. The predictions were performed every 12 hours.

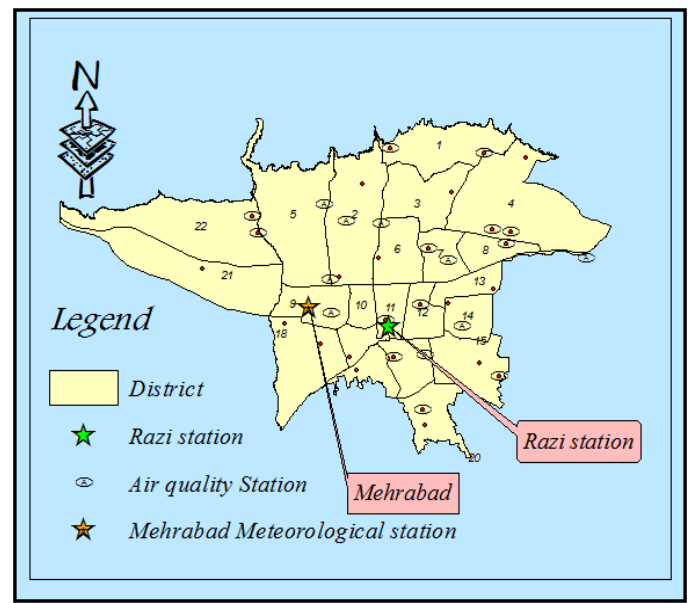

Figure 1. Tehran city, Distribution of air quality stations and Mehrabad meteorological station

\section{TEMPERATURE INVERSION AND IT'S EFFECT ON POLLUTANT CONCENTRATION}

Normally and physically, being heavy would lead to movement the warm air upward and the cold air downward. In the early morning, when the sun rays, the earth layers become warmer of the lower layers due to the sun rays hitting. By starting the day and beginning of human activities and fuel consumption, the surface air temperature becomes high.

When the warm air moves upward during ascent or displacement by wind, it strikes with isothermal layer of air in the upper atmosphere that is composed of solar radiation. This occurrence prevents air to move upward. Thereby, forming a coverage surface above the town would be resulted that causes the produced pollutants remain in the surface layer and don't move upward even by passing of time. This would be happened due to human-caused activity and air-trapped under this layer. These pollutants are not involved in creation of this phenomenon. Air temperature in troposphere layer usually decreases by height. Sometimes, in layers, temperature increases with the height, called a "temperature inversion". Temperature inversion is sometimes seen from upwards ground level. It is called "surface inversion" and sometimes it can be seen in a layer above the ground that is "upper inversion". Although the role of human in polluting the atmosphere is effectively, but, nature with the inversion phenomenon intensifies the issue. Because, when this phenomenon occurs, cold weather and pollutant is at low height. Finally, it makes the climate stable. Thus, the chance of air conditioning is not possible on the days that are associated with this phenomenon since performance and density levels differ greatly.

One of the parameters that can demonstrate this distinction very well is the inversion strength and its unit is $0^{c} / 100 \mathrm{~m}$. To find the inversion strength in the surface layer as the effective concentration at ground level concentrations, Eq. (1) could be used (Stefanie, 2008):

Inversion strength $=\frac{d t}{d h}$

where $d t$ is the difference between the peak of temperature inversion and ambient temperature, and $d h$ is inversion height. The inversion strength values are interpreted as shown in Table 1.

\begin{tabular}{|l|c|c|}
\hline Stability classification & $\begin{array}{c}\text { Stability } \\
\text { category }\end{array}$ & $\begin{array}{c}\text { Range of vertical } \\
\text { temperature gradient } \\
\left({ }^{\circ} \mathrm{C} / 100 \mathrm{~m}\right)\end{array}$ \\
\hline Extremely unstable & $\mathrm{A}$ & $\mathrm{DT} / \mathrm{DZ}<-1.9$ \\
\hline Moderately unstable & $\mathrm{B}$ & $-1.9<=\mathrm{DT} / \mathrm{DZ}<-1.7$ \\
\hline Slightly unstable & $\mathrm{C}$ & $-1.7<=\mathrm{DT} / \mathrm{DZ}<-1.5$ \\
\hline Neutral & $\mathrm{D}$ & $-1.5<=\mathrm{DT} / \mathrm{DZ}<-0.5$ \\
\hline Slightly stable & $\mathrm{E}$ & $-0.5<=\mathrm{DT} / \mathrm{DZ}<1.5$ \\
\hline Moderately stable & $\mathrm{F}$ & $1.5<=\mathrm{DT} / \mathrm{DZ}<4$ \\
\hline Extremely stable & $\mathrm{G}$ & $4<=\mathrm{DT} / \mathrm{DZ}$ \\
\hline
\end{tabular}

Table 1.inversion strength ranges\& interpreted

It should be noted that in Tehran due to geographical location and climate, 2/3 times a year we are facing inversion. This problem is more severe during the cold season and causing pollution at ground surface. Therefore, it is very important and cannot be ignored in the air pollution forecasting.

\section{THE PROPOSED METHODOLOGY}

We propose an approach based on integrating statistical information and ANN to predict the PM2.5 concentration. The schematic structure of the proposed method is show in Figure 2. At first, the data was prepared for importing into the ANN (Section 5.1). Then the network was trained (Section 5.2), and finally, the model performance was calculated by statistical parameters (Section 5.3).

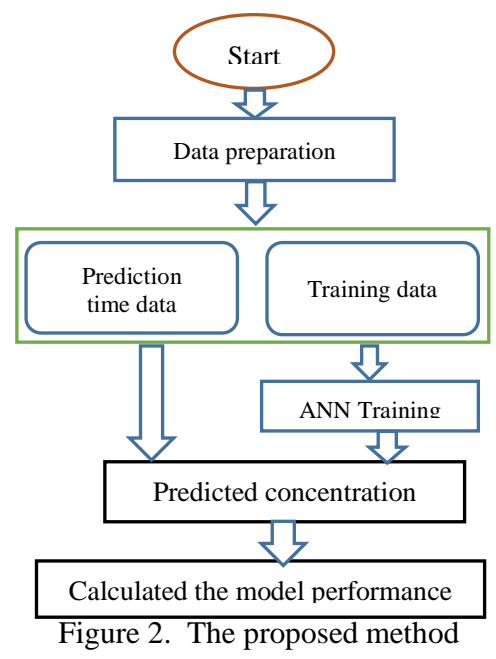

\subsection{Data preparation}

In this study, the input data are:

- $\quad$ PM2.5 concentration $\left(\mu \mathrm{g} / \mathrm{m}^{3}\right)$

- Temperature $\left({ }^{\circ} \mathrm{C}\right)$

- Wind speed $(\mathrm{m} / \mathrm{s})$ 
- Wind direction (degree from north)

- $\quad$ Relative humidity $(\%)$

- Cloud cover (Octa)

- Temperature inversion strength $\left({ }^{\circ} \mathrm{C} / 100 \mathrm{~m}\right)$

This data were prepared for both years 2012 and 2013 in two times of the day (0\&12 UTC).

Figures 3-16 show the values of the input parameters for both years 2012 and 2013.

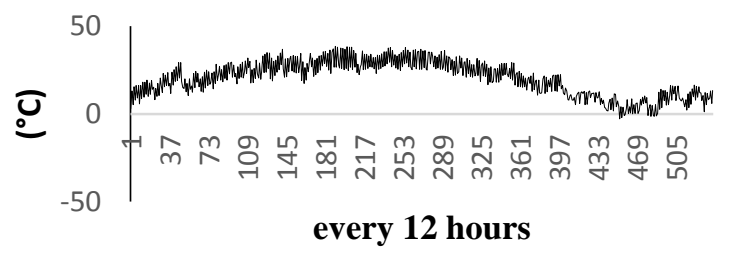

Figure 3. Temperature for every $12^{h}$ in 2012

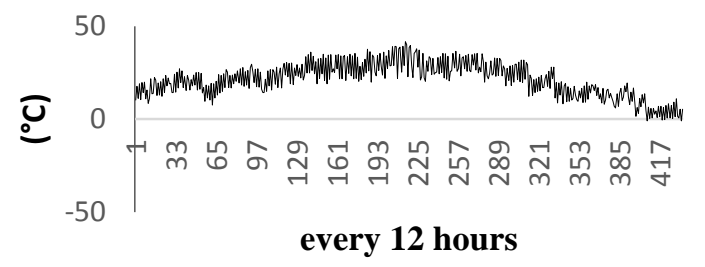

Figure 4. Temperature for every $12^{h}$ in 2013

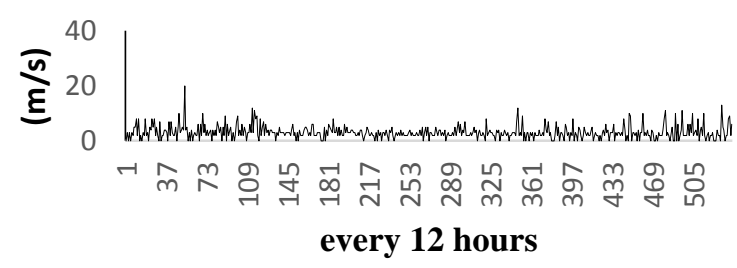

Figure 5.Wind Speed for every $12^{h}$ in 2012

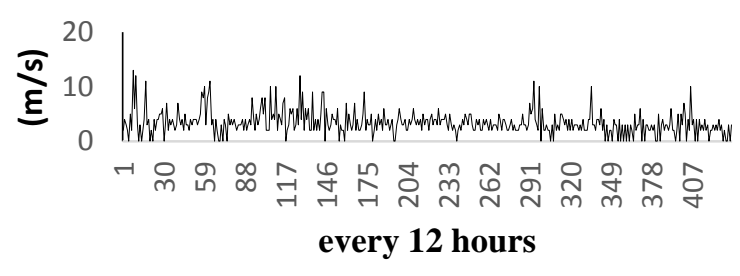

Figure 6.Wind Speed for every $12^{h}$ in 2013

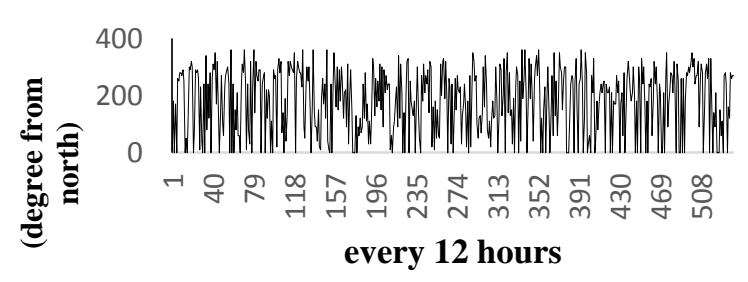

Figure 7.Wind Direction for every $12^{h}$ in 2012

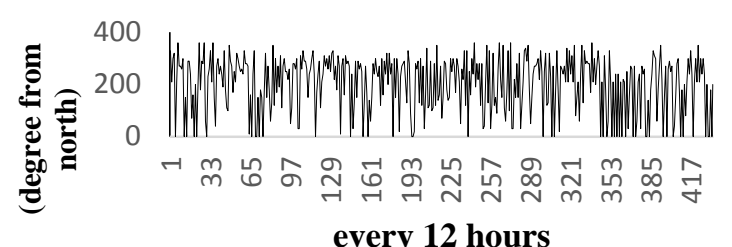

Figure 8.Wind Direction for every $12^{h}$ in 2013

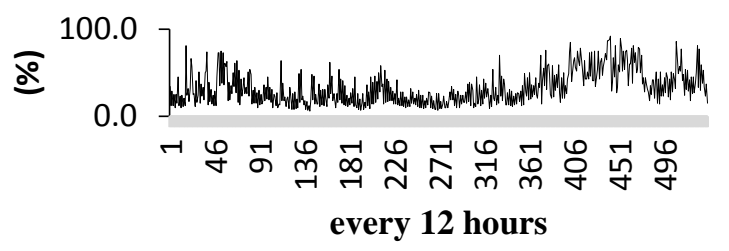

Figure 9 .Relative Humidity for every $12^{h}$ in 2012

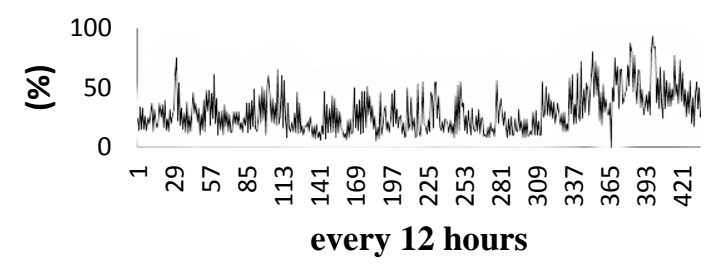

Figure 10.Relative Humidity for every $12^{h}$ in 2013

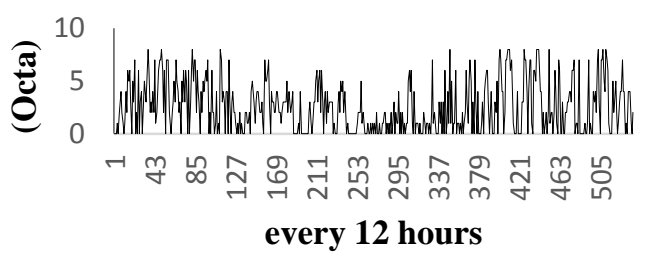

Figure 11. Cloud Cover for every $12^{h}$ in 2012

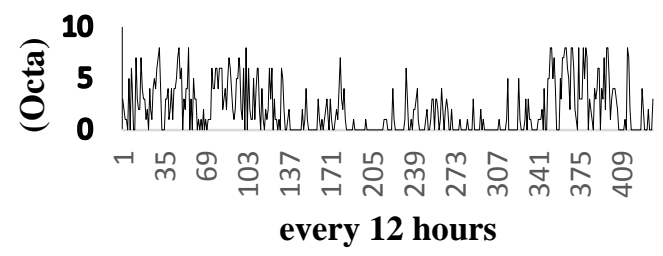

Figure 12. Cloud Cover for every $12^{h}$ in 2013

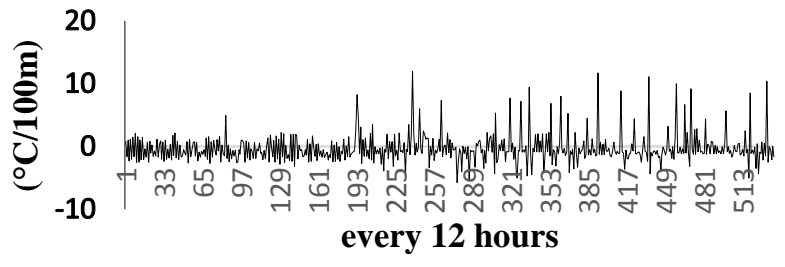

Figure 13. Temperature inversion for every $12^{h}$ in 2012 


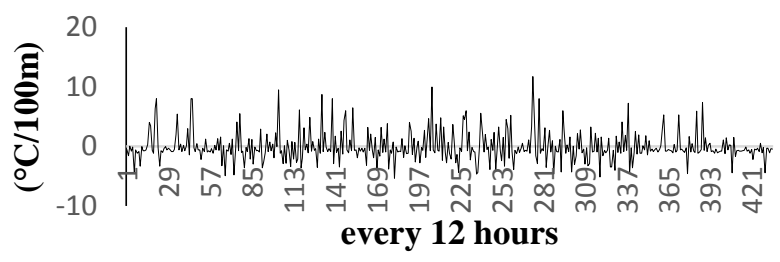

Figure 14. Temperature inversion for every $12^{h}$ in 2013

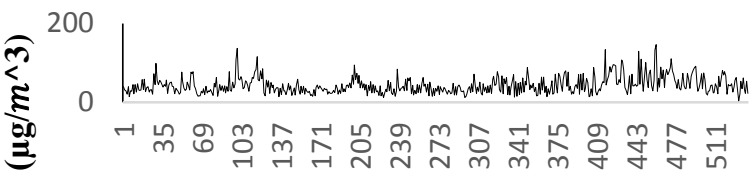

$$
\begin{aligned}
& \text { every } 12 \text { hours }
\end{aligned}
$$

Figure 15. PM2.5 Density for every $12^{h}$ in 2012

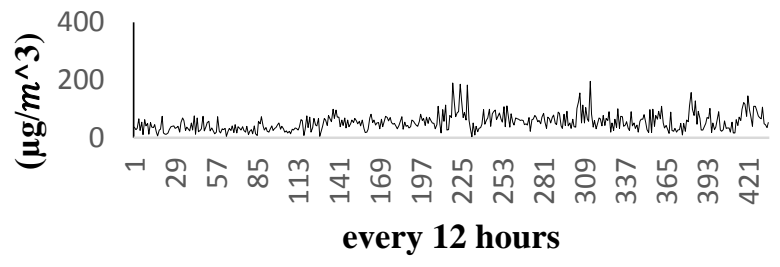

Figure 16. PM2.5 Density for every $12^{h}$ in 2013

To produce a temperature inversion data, skew-t diagram of 1400 and 1400 Data Table of upper atmosphere information were determined by Mehrabad Station. The rate of changes of temperature up to $850 \mathrm{hPa}$ layer was calculated for each 12 hours. The rate of the temperature of the formula described in Eq. (1) points to "inversion strength" at the days the inversion would be happened, and in contrary, indicated to the amount of "air follow rate" at the days the inversion does not occur.

Each input has its own unit and also has different range value. In order to optimize the network training phase, the values of climate and pollution were normalized in the range [0 1 1] before entering the network training phase. Normalizing input data avoids weights numerically being too big or too small in the computation.

\subsection{The proposed neural network}

Artificial neural networks are one of the most popular methods of artificial intelligence that mimic mathematical model of the human brain. Neural networks are usually trained with training data. They enable new connections, new functions or new models to explore. And because of the above properties have been widely used. One of the most effective arrangement proposed for use in modelling is the multilayer perceptron or briefly MLP that is formed by an input layer, one or more hidden layers and an output layer (Figure 17).

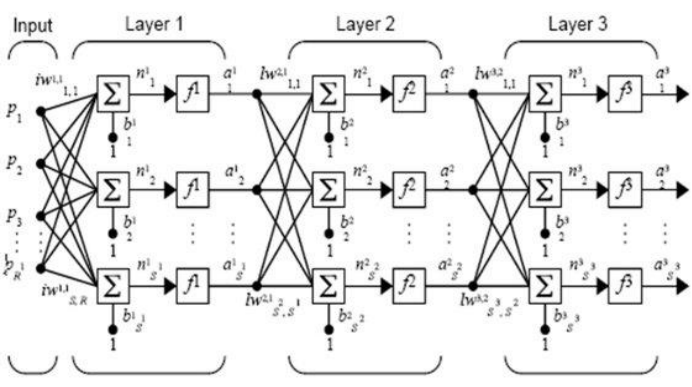

Figure 17. The MLP network structure

The relation between inputs and outputs of a neuron is shown as

$$
\text { Where } \quad \begin{aligned}
& \boldsymbol{p}=\text { inputs } \\
& \boldsymbol{w}=\text { weights } \\
& \boldsymbol{b}=\text { a bias for a single neuron } \\
& \boldsymbol{f}=\text { transfer function } \\
& \boldsymbol{a}=\text { the output }
\end{aligned}
$$

The weights would be updated by the structure shown in Figure 18

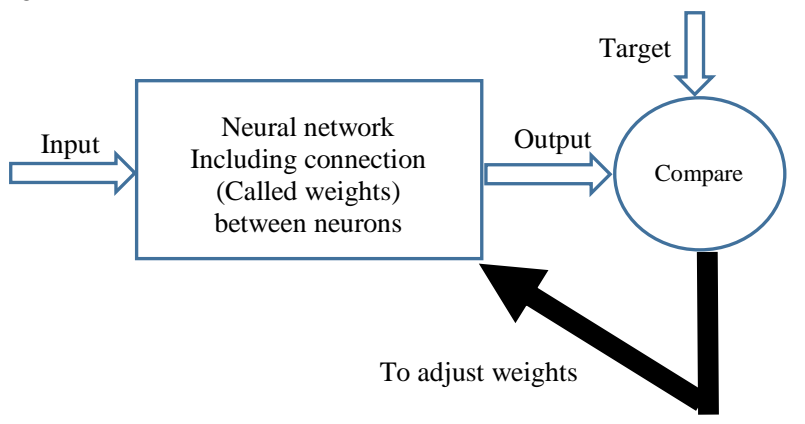

Figure 18. The MLP network structure

In this structure, all the neurons in one layer are connected to all neurons in the next layer. These networks are able to select the appropriate number of layers and neurons. In recent decades, these networks have been greatly used in air quality forecasting (Bodaghpour, 2009). In this study, the MLP neural network was used to predict the concentration of PM2.5 emissions. Figure 19 shows the proposed MLP structure with one hidden layer which includes 10 neurons, as well as single output as PM2.5.

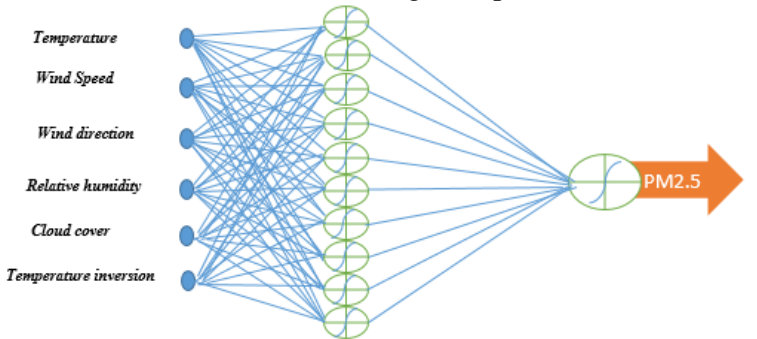

Figur19. The proposed MLP

\subsection{Statistical parameters}

In this study, two statistical parameters were used to analyse the performance of the model. The first parameter is Root Mean Squared Error (RMSE) that represents the measure of the difference between the values predicted by a model and the values actually observed from the environment that is being modelled, and expresses the predictive power of the model.

Another parameter is the coefficient of determination $\left(\mathrm{R}^{2}\right)$ which indicates the goodness of fit of a model (Ahmad Zia et al, 2013). 
This parameter is between 0 and 1, in a way higher values show that the model was fitted well. This parameter is calculated using the Eq. (3).

$$
R^{2}=\left(\frac{\sum_{i=1}^{n}\left(p_{i}-\bar{p}\right)\left(o_{i}-\bar{o}\right)}{n \cdot s_{p r e d} \cdot s_{o b s}}\right)^{2}
$$

Where $\quad p_{i}=$ the $\mathrm{i}^{\text {th }}$ predicted concentration

$\bar{p}=$ the average of predicted concentration

$o_{i}=$ the $\mathrm{i}^{\text {th }}$ observation concentration

$\bar{o}=$ the average of observation concentration

$s_{\text {pred }}=$ the standard deviation of predicted values

$s_{o b s}=$ the standard deviation of the observed values

\section{RESULTS}

In this study, hourly input parameters that we want to predict the concentration in that in addition to the training data were set up in the neural network learning algorithm. After the network was trained, the concentration in that time was predicted. In this study, the parameters of the proposed MLP neural network were set according to Table 3 .

\begin{tabular}{|l|c|}
\hline \multicolumn{2}{|l|}{ The propose neural network properties } \\
\hline Number of layers & $r$ \\
\hline Training algorithm & Levenberg-Marquardt (LM) \\
\hline Hidden layer transfer function & tangent sigmoid \\
\hline Output layer transfer function & tangent sigmoid \\
\hline Number of neurons in the hidden layer & 10 \\
\hline
\end{tabular}

Table 3.Parameters of the proposed MLP

PM2.5 concentration was predicted for 72-hour and the estimated results are shown in Table 4.

\begin{tabular}{|l|c|c|c|}
\hline Time & Performance & $\mathrm{R}^{2}$ & RMSE \\
\hline Next $12^{h}$ & 0.002 & 0.7942 & 9.088 \\
\hline Next two $12^{h}$ & 0.002 & 0.7714 & 8.421 \\
\hline Next three $12^{h}$ & 0.002 & 0.7114 & 10.363 \\
\hline Next four $12^{h}$ & 0.002 & 0.7343 & 12.616 \\
\hline Next five $12^{h}$ & 0.002 & 0.5756 & 9.969 \\
\hline Next six $12^{h}$ & 0.002 & 0.6340 & 12.010 \\
\hline
\end{tabular}

Table 4. The estimated results

The Figure 20 illustrates the performance of the proposed MLP neural network.

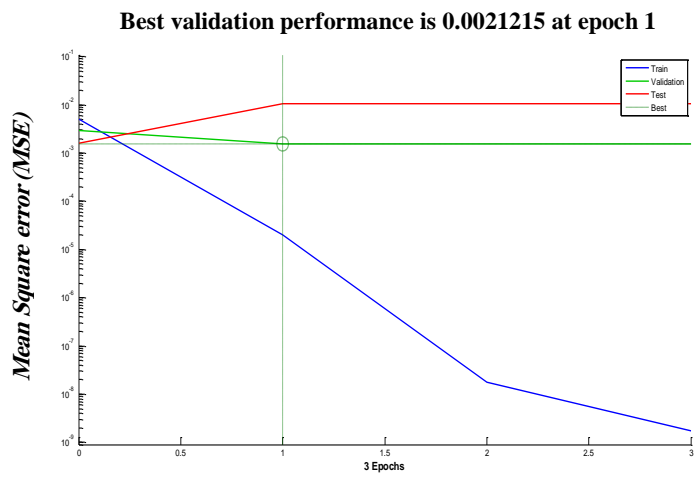

Figure 20. The performance of the proposed MLP neural network training

\section{CONCLUSIONS}

In this paper, attempts were made on predicting the 72-hour PM2.5 concentration data by proposing a MLP neural network for Razi Station.

In this study, based on the goodness of fit parameter, i.e., $R^{2}$, the correlation coefficient between actual and predicted values was concluded. The $R^{2}$ values were between 0.57 and 0.79 that indicated a well-fitting. Also, by applying temperature inversion strength as one of the input parameters of the proposed MLP neural network, the prediction was improved.

Therefore, we can conclude that the proposed MLP neural network is a powerful tool in predicting the performance of these model of crisis management in Tehran air quality and can assist managers in critical conditions.

\section{REFERENCES}

1. Bodaghpour et al. 2009, Prediction of air pollution due to traffic by means of Artificial Neural Network. World applied Sciences Journal, 7, pp. 156-161.

2. Ibarra-Berastegi et al. 2008, from diagnosis to prognosis for forecasting air pollution using neural networks: Air pollution monitoring in Bilbao. Environmental Modelling \& Software, 23, pp. 622637.

3. Nejadkoorki, F., Baroutian, S. 2012, Forecasting Extreme PM10 Concentrations Using Artificial Neural Networks. Int. J. Environ. Res., 6(1), pp. 277-284.

4. Menhaj, m., 2005. Fundamentals of Artificial Neural Networks. Amir Kabir publication, Iran,

5. Panjekoobi,p., 2011. Analysis of temperature inversion and its effect on air pollution in Tehran, A thesis Presented to the Faculty of the University of Tehran, Tehran, pp.29-60.

6. Ahmad Zia et al., 2013. Future daily PM10 concentrations prediction by combining regression models and feed forward back propagation models with principle component analysis (PCA). Atmospheric Environment 77, pp. 621-630.

7. Stefanie M. Bourne., 2008. A climate perspective of observed and modeled surface-based temperature inversions in Alaska, A thesis Presented to the Faculty of the University of Alaska Fairbanks Alaska, pp. 1844.

8. Hatefi, m., 2007. A GIS-based Air Pollution Modeling in Tehran. Environmental Informatics Archives, Volume, 5, pp. 557- 566.

9. Surendra Roy, .2012. Prediction of Particulate Matter Concentrations Using Artificial Neural Network. Resources and Environment, 2(2), pp. 30-36.

10. Anikender Kumar, P, .2011. Forecasting of daily air quality index in Delhi. Science of the Total Environment, 409, pp. 5517-5523.

11. Khazaei, p, .2012. Comparison of two modeling methods using Neuro Fuzzy system to predicted pollutant concentrations of carbon monoxide. Ecology, 4, pp. 29-44. 